$\mathrm{X}$.

\title{
Sitzungsbericht der oto-laryngologischen Sektion des Warschauer ärztlichen Vereins. (Medycyna 1906.)
}

\begin{abstract}
1. Garanowski spricht uber den gegenwärtigen Stand der Lehre der Otosklerose.

In der Diskussion hebt Meyerson hervor, daB, obgleich die Statistik noch zu keinem bestimmten Resultate gefuhrt hat, er doch den Eindruck habe, daß die Syphilis bei dieser Krankheit keine wesentliche ātiologisehe Rolle spielt. Da manche Fälle dieser Krankheit mit rein katarrhalischen, besserungsfähigen Yeränderungen kompliziert sind, ist die Prognose nicht
\end{abstract} durchaus ungünstig zu stellen.

Srebrny erwähnt einen von ihm beobachteten Fall von Otosklerose bei einem 15 jăhrigen Patienten. Auch er ist der Ansicht, daß Syphilis hier keine ätiologische Bedeutung besitze, dagegen hat er oft diese Krankheit bei Arthritikern angetroffen. Zur Differenzierung der Krankheit von den katarrhalischen Veränderungen dient der Effekt der Lufteintreibung. Ist dieser positiv, so kann man dem Patienten Besserung versprechen. $\mathrm{S}$. hält es nicht für richtig, dem Patienten die Hoffnungslosigkeit seines Zustandes zu offenbaren und so jeden Funken einer Hoffunng in ihm zu verlöschen.

Ch orazycki hat Koinzidenz dieser Krankheit mit ubermăbiger Weite der Nasengänge beobachtet und glaubt einen Zusammenhang zwischen diesen Afrektionen annehmen zu sollen, stöbt aber dabei auf Widerspruch bei anderen Rednern.

Heimann hält es auch für zu grausam, dem Patienten jede Hoffnung zu nehmen. Als eines der frühesten Symptome der sklerose hat H. das Auftreten einer vorderen Trommelfellfalte beoliachtet.

Guranowski erklärt, dab er die wahre Aufklärung des Kranken über seinen Zustand für notwendig erachte, um ihn von der Wabl eines Berufes abzuhalten oder inn zur Anfgabe eines solchen zu bewegen, der ein gutes Gehör erforderlich macht.

2. Heimann bespricht anläßlich eines von ihm beobachteten einschlägigen Falles den Verlauf der Mittelohrentzündung bei Diabetes. Bei Diabetes pflegen zwei Formen von Ohrenkrankheiten vorzukommen, 1. selten eine Affektion des inneren Ohres, 2. häufiger eine eitrige Mittelohrentzündung, der man den Charakter einer besonderen Bösartigkeit zuzuschreiben versuchte. H. konnte in seinen Fallen keinen Unterschied im Verlaufe dieser Krankheit bei Diabetes im Vergleiche zu den sonstigen Fällen beobachten. Bei einem 80 jährigen Mame mit $31 / 2 \%$ Zucker im Harn fand $\mathrm{H}$. in der 4. Woche einer akuten Media geringe Eiternng im Ohre, unbedeutende Schmerzhaftigkeit am Proc. mast., Temp. 39.5 ${ }^{\circ}$. Aber sehon nach einer Woche war die Hitze verschwunden, die Eitermng versiegt, das Trommelfell geschlossen. Gehör für Taschenubr $=0$, für die Sprache $=1 \frac{1}{2}$ Meter. Nur klagte der Patient noch über grolso Schwäche. Am Abend desselben Tages Temperatursteigerung ohne Schüttelfrost. Zwei Wochen später plötzlicher Bewußtseinsverlust mit dem Bilde einer Meningitis ohne vorausgegangene Erscheinungen. 18 Stunden später Exitus. H. meint, daß die Schwäche, über die der Patient klagte, und die geringe Hör- 
fähigkeit auf eine etwa bestehende Komplikation hätte aufmerksam machen können.

Chorazycki hat einen 23 jăhrigen Diabetiker mit einer akuten eitrigen Mittelohrentzündung beobachtet, der sich nach der Parazentese wohl befand, am folgenden Tage sich schlecht fühlte, schwachen Puls aufwies und tags darauf verschied.

Heiman führt den Fall einer 72 jährigen Frau an, die auf beiden Ohren ertaubte. Die Untersuchung ergab Labyrinthtaubbeit und Diabetes. Nach einem 2 wöchentlichen Gebrauche von Karlsbader Wasser kehrte das Gehör zurück.

Sok olowski teilt die Ansicht H's., daß die Otitis media bei Diabetes sich nicht von der gewöhnlichen Mittelohrentzündung unterscheide und auch die Trockenheit des Halses, die man dem Diabetes zuschrieb, ist nicht charakteristisch, und rührt nur von dem oft gesteigerten Durstgefühl her; hingegen sieht man oft Diabetes ohne Pharyngitis sicca. Auch für blutige Eingriffe bilde Diabetes keine Kontraindikation, wie man lange Zeit glaubte. Srebrny hat selbst eine Trepanation des Warzenfortsatzes bei einem an Diabetes leidenden Patienten beobachtet, der von jeder Komplikation frei blieb. Hingegen hat er in einem Fall nach der Inzision eines Furunkels des äußeren Gehörganges einige Wochen mit der Krankheit zu kämpfen, bis es ihm gelang, Heilung herbeizuführen.

3. Zebrowski, M. Über die Heilbarkeit und operative Behandlung otitischer Pyaemie. Auf Grund der von ihm beobachteten und operierten Fälle kommt Z. zu folgenden Konklusionen: 1. Die otitische Pyaemie verläuft unter verschiedenen Formen und die operativen Eingriffe müssen je nach der Intensität der Krankheit und den während der Trepanation gefundenen pathologisch - anatomischen Veränderungen modifiziert werden. 2. Die vollständige Elimination des Krankheitsherdes aus dem Schläfenbein und die Bloßlegung des Sinus transversus reichen oft allein vollkommen zur Heilung der Pyaemie aus. 3. Mehrwertiges Antistreptokokkenserum kann manchmal auf den postoperativen Verlauf otitischer Pyaemie einen sehr günstigen Einfluf ausüben. r. Fehlen von Schmerzhaftigkeit des Warzenfortsatzes bei eitrigen Prozessen des Mittelohres kann keine Kontraindikation gegen einen operativen Eingriff bilden. 5. Das Erscheinen von Symptomen einer Phlebitis des Sinus cavernosus ist als ein Zeichen des nahen Exitus, jedweder Versuch einer operativen Eröffnung dieses Sinus als unstatthaft anzusehen.

Heiman findet in diesem Vortrag eine Bestätigung seiner Beobachtung, daß otitische Pyaemien mit Metastasen in den peripheren Organen (Gelenke, Muskeln usw.) eine bessere Prognose gestatten, als jene mit Metastasen in den inneren Organen. Die von Grunert und Vos s praktizierte Eröffnung des Bulbus venae jugularis behufs Entfernung von Thromben aus demselben ergab ihm bis jetzt keine befriedigenden Resultate.

Spira (Krakau). 